\title{
Analysis of Screen Channel LAD Bubble Point Tests in Liquid Oxygen at Elevated Temperature
}

\author{
Jason Hartwig ${ }^{1}$ \\ ASRC Aerospace, Cleveland, $\mathrm{OH}, 44135, \mathrm{USA}$ \\ John McQuillen ${ }^{2}$ \\ NASA Glenn Research Center at Lewis Field, Cleveland, OH, 44135, USA
}

\begin{abstract}
The purpose of this paper is to examine the key parameters that affect the bubble point pressure for screen channel Liquid Acquisition Devices in cryogenic liquid oxygen at elevated pressures and temperatures. An in depth analysis of the effect of varying temperature, pressure, and pressurization gas on bubble point is presented. Testing of a $200 \times 1400$ and $325 \times 2300$ Dutch Twill screen sample was conducted in the Cryogenics Components Lab 7 facility at the NASA Glenn Research Center in Cleveland, Ohio. Test conditions ranged from 92 to $130 \mathrm{~K}$ and $0.138-1.79 \mathrm{MPa}$. Bubble point is shown to be a strong function of temperature with a secondary dependence on pressure. The pressure dependence is believed to be a function of the amount of evaporation and condensation occurring at the screen. Good agreement exists between data and theory for normally saturated liquid but the model generally under predicts the bubble point in subcooled liquid. Better correlation with the data is obtained by using the liquid temperature at the screen to determine surface tension of the fluid, as opposed to the bulk liquid temperature.
\end{abstract}

\section{Nomenclature}

$\begin{array}{ll}D_{p} & =\text { pore diameter }[\mu \mathrm{m}] \\ T_{c} & =\text { Critical temperature }[\mathrm{K}] \\ \Delta P_{B P} & \left.=\text { Bubble point pressure [in } \mathrm{H}_{2} \mathrm{O}\right] \\ \Delta P_{\text {dynamic }} & =\text { Pressure loss for mass accumulation along channel length } \\ \Delta P_{\text {friction }} & =\text { Friction pressure loss for flow along channel } \\ \Delta P_{F T S} & =\text { Pressure loss for flow through screen } \\ \Delta P_{\text {hydrostatic }} & =\text { Hydrostatic pressure } \\ \Delta P_{\text {Other }} & =\text { Other sources of pressure fluctuations and differences } \\ \sigma & =\text { Surface tension }\left[\mathrm{J} / \mathrm{m}^{2}\right] \\ \theta_{c} & =\text { Contact angle }\end{array}$

\section{Introduction}

GRAVITY affects the positioning of the liquid and vapor phases within a propellant tank. When feeding propellant from the storage tank to a spacecraft engine in reduced gravity, it is necessary to transport only the single phase liquid to ensure the efficient thermal conditioning and the safe and stable operation of the engine. One device used to collect liquid from within the propellant tank in varying thermal and gravitational environments is the screen channel Liquid Acquisition Device (LAD). These LADs rely on capillary flow and surface tension forces within micron sized pores to wick fluid into a channel, and also to maintain a barrier to gas ingestion as

\footnotetext{
${ }^{1}$ Research Aerospace Engineer, Propellants and Propulsion Branch, 21000 Brookpark Road, MS 500-1, Cleveland, $\mathrm{OH}$ 44135, and AIAA Member.

2 Senior Aerospace Engineer, Fluid Physics and Transport Branch, 21000 Brookpark Road, MS 77-5, Cleveland, $\mathrm{OH}$ 44135, Non-Member.
} 
liquid is expelled from a tank. Screen channel LADs tend to follow the contours of the tank, with various channels or "gallery arms", which extend along the entire tank to ensure total communication at all times between propellant and tank outlet during outflow. The side that faces the wall has millions of micron sized openings which serve two purposes: to act as a barrier to vapor ingestion and to allow liquid to be wicked into the pores during outflow.

Screen channel LADs are primarily characterized by the geometry, size, and number of pores, which is compactly expressed as the screen weave. The screen weave refers to the number of wires per inch in each direction and the weave pattern, or the specific pattern used during the screen manufacturing process. Figure 1 displays a commonly used 200x1400 (200 warp wires and 1400 weft wires) Dutch Twill screen style. In this design, each weft wire passes over two warp wires before going under the next warp wire. The screen weave is an important parameter affecting the choice of screen channel LAD since certain weaves of wires are capable of producing much finer pore sizes than other weaves. In general, finer screen meshes are desirable to ensure adequate resistance to vapor ingestion. However, they tend to generate a large pressure loss through the screen during propellant outflow. Finer mesh screen styles have smaller pores, which also make them susceptible to clogging if impurities exist within the propellant.

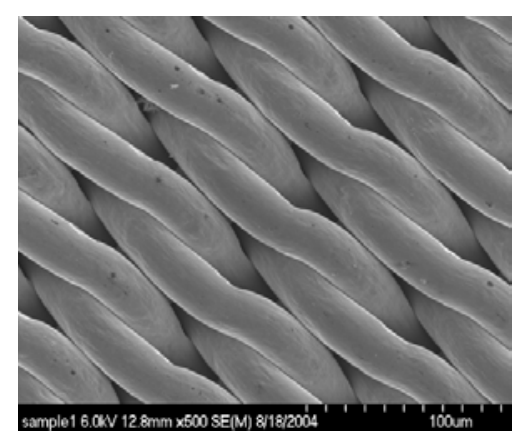

Figure 1: SEM Photograph of a 200x1400 Screen Channel LAD Sample

\section{The Bubble Point}

The primary method for characterizing screen performance is to measure the bubble point, or the differential pressure required for vapor or gas bubbles to overcome liquid surface tension forces and penetrate the screen's pores. For a normally saturated fluid, the bubble point is expressed as follows:

$$
\Delta P_{B P}=\frac{4 \sigma \cos \theta_{C}}{D_{P}}
$$

Thus the bubble point is a function of the surface tension of the liquid $\sigma$, the contact angle between solid screen pore and liquid, $\theta_{c}$, and the "effective" pore diameter of the screen, $D_{P}$. It is assumed that the contact angle between all cryogens and stainless steel is zero such that $\cos \theta_{c}=1$. Since it is quite difficult to define a pore diameter for Dutch Twill screens, the general practice is to measure the bubble point of the screen sample in a standard reference fluid, such as isopropyl alcohol (IPA), and then use similitude to determine the average pore size for all other liquids. Due to small variations in screen manufacturing processing, this method only works well if the exact same screen sample is tested in these fluids.

As illustrated in Figure 1, for a full screen channel LAD system inside a propellant tank, the total pressure drop may be expressed as:

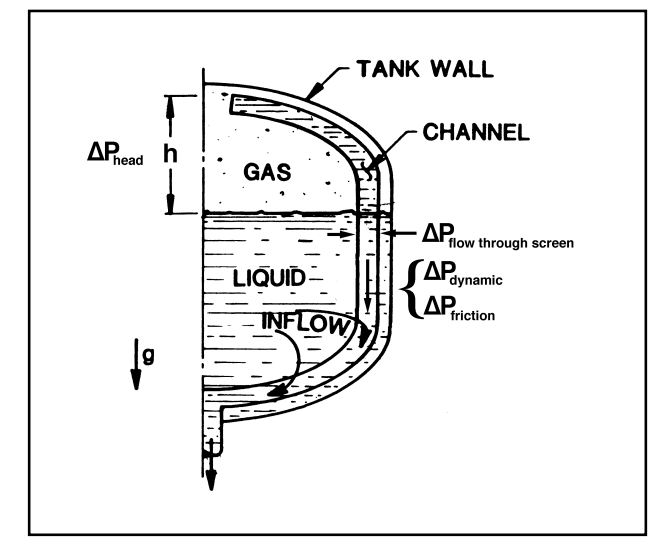

Figure 2: Illustration of Pressure Losses in a Screen Channel LAD (reprinted from Van Dyke 1998)

$$
\Delta P_{\text {total }}=\Delta P_{\text {hydrostatic }}+\Delta P_{\text {FTS }}+\Delta P_{\text {friction }}+\Delta P_{\text {dynamic }}+\Delta P_{\text {other }}
$$

where $\Delta P_{\text {hydrostatic }}$ is the hydrostatic pressure on the LAD screen, $\Delta P_{F T S}$ is the pressure drop across the screen, $\Delta P_{\text {friction }}$ is the frictional losses down the LAD channel, and $\Delta P_{\text {dynamic }}$ is the pressure drop due to flow in the channel. There may be several other pressure loss terms due to transients, vibrations, and propellant sloshing, but these are neglected for the time being. In a flight propellant tank, this total pressure loss must be less than the bubble point pressure to prevent vapor ingestion into the outflow line and to the engine. Therefore for a given cryogenic propellant and screen style, for a given operating range of flow rate, temperature, and pressure, the bubble point serves as an upper limit on the total allowable pressure loss for that system. For a given LAD screen style, the bubble point is readily measurable and easily comparable with theoretical predictions and therefore serves as the primary performance parameter for screen channel LADs. 


\section{Previously Reported Bubble Points}

Historically, screens and screen channel LADs are well characterized for storable propellants (propellants that exist as a liquid at room temperature) and for cryogens at or near the saturated state at the low pressures typical of pumpfed engines. Previous screen channel LAD bubble point tests have been conducted in liquid hydrogen $\left(\mathrm{LH}_{2}\right)$, liquid nitrogen $\left(\mathrm{LN}_{2}\right)$, liquid oxygen ( $\left.\mathrm{LOX}\right)$, and liquid methane $\left(\mathrm{LCH}_{4}\right)$ at saturation pressures near standard conditions. Figure 3 summarizes previous measurements for a 325x2300 Dutch Twill screen sample for these cryogenic liquids, along with reference bubble point values in IPA as reported by Paynter ${ }^{1}(1973)$, Cady $(1973)^{2}$, Cady $(1975)^{3}$, Cady $(1977)^{4}$, Chato and Kudlac (2002) ${ }^{5}$, Kudlac and Jurns (2005) $)^{6}$, and Jurns et. al (2007) ${ }^{7}$, along with a prediction line based on Equation 1. As shown, good agreement exists between previous measurements and the bubble point prediction based on simple surface tension theory.

While Equation 1 correlates reasonably well when using the bulk liquid temperature to predict the bubble point for conditions near atmospheric pressure, there is deviation when the liquid is in a subcooled state (liquid temperature at the screen is lower than the saturation temperature based on the pressure at the screen). Initial efforts to predict the bubble point for a subcooled liquid based on the bulk liquid temperature were attempted by Jurns et. al (2007) for $\mathrm{LCH}_{4}$ subcooled slightly below atmospheric conditions. The original

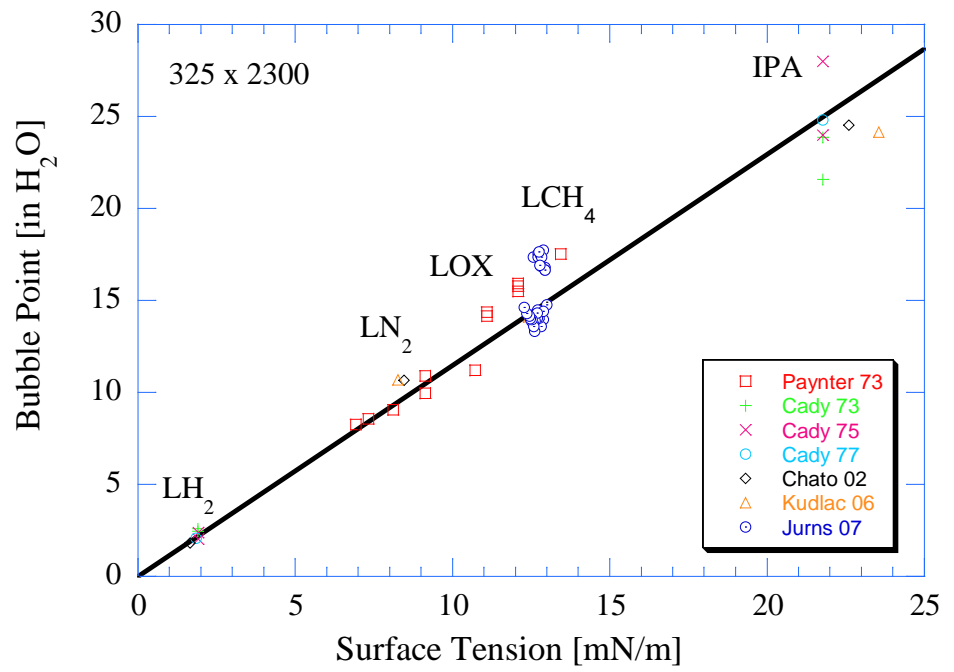

Figure 3: Previously Reported Bubble Point Values for the 325x2300 Dutch Twill Screen bubble point equation was modified by taking into account the fluid viscosity and density using a relationship developed by Bretherton ${ }^{8}$ for the slow motion of an elongated bubble through a capillary tube. While the analysis did correlate well for subcooled liquid methane, Jurns and McQuillen ${ }^{9}$ found that this correction term did not hold when applied to results for subcooled liquid oxygen over the same small temperature range.

\section{Motivation for These Tests}

Recently, there has been a growing desire to develop technology to enable higher pressure fed cryogenic propellant engines to take advantage of higher engine performance at higher operating pressures. Rather than extrapolate screen channel LAD performance at these elevated conditions, it was determined that bubble point measurements needed to be conducted at or near these operating conditions, since there was up to a $9 \%$ discrepancy in surface tension data for liquid oxygen from different published sources (Roder and Weber) ${ }^{10}$. To enable future higher pressure-fed engine capabilities for longer duration space missions, such as the proposed Lunar and Martian ascent stages or Earth Departure Stage (EDS), ground tests are first required to fully characterize these LADs over a wide range of thermodynamic conditions. Given that some design reference missions proposed the use of pressurized cryogenic propellant tanks, and due to the paucity of data at these conditions, the Cryogenic Fluid Management (CFM) program at NASA Glenn Research Center (GRC) opted to pursue experiments to measure the bubble point for LOX at elevated pressures and temperatures representative of the proposed Lunar Ascent Stage for Altair.

Screen channel LAD performance in high pressure propellant tanks may be affected by the degree of propellant subcooling and type of gas used during pressurization or liquid expulsion. The authors ensured bubble point data was collected over the widest possible range of thermal conditions inside a LOX propellant tank, consistent with the limitations of the test hardware. Therefore, the purpose of this paper is to conduct an in-depth analysis on these recently concluded bubble point tests to understand each of the parameters that affect LAD performance in an elevated pressure environment. 


\section{Test Description}

Testing was conducted in the Cryogenics Components Lab 7 (CCL-7) facility at GRC in Cleveland, Ohio. Two LAD screen samples were tested, a $200 \mathrm{x}$ 1400 and $325 \times 2300$ Dutch Twill screen. The thickness of the $200 \times 1400$ and $325 \mathrm{x}$ 2300 screen samples was $152 \mu \mathrm{m}$ and 89 $\mu \mathrm{m}$, respectively. Using IPA, the effective pore diameter for these particular samples was previously measured as $20.0 \mu \mathrm{m}$ and $14.0 \mu \mathrm{m}$ for the $200 \times 1400$ and $325 \times$ 2300 screens, respectively. Screen samples were each mounted onto a $2.38 \mathrm{~cm}$ tall, $5.08 \mathrm{~cm}(2 ")$ outer diameter (OD) cup, which was mounted inside a $3.55 \mathrm{MPa}$ (500 psig) rated, $15 \mathrm{~cm}(6 ")$ OD, $33.65 \mathrm{~cm}$ $(13.25 ")$ high $6000 \mathrm{~cm}^{3}$, optically accessible CFM test tank, which was mounted inside a $0.229 \mathrm{~m}^{3}\left(8.1 \mathrm{ft}^{3}\right)$ receiver dewar as shown in Figure 4.

Bubble point tests were conducted in an inverted fashion with the liquid on top and gas on the bottom of the screen in order to precisely control the liquid head pressure and to allow bubbles to naturally rise to the tank ullage space after screen breakdown. The purpose of the cup assembly was to pressurize the LAD screen with gas from beneath to create a liquid vapor interface within the screen pores. The purpose of the

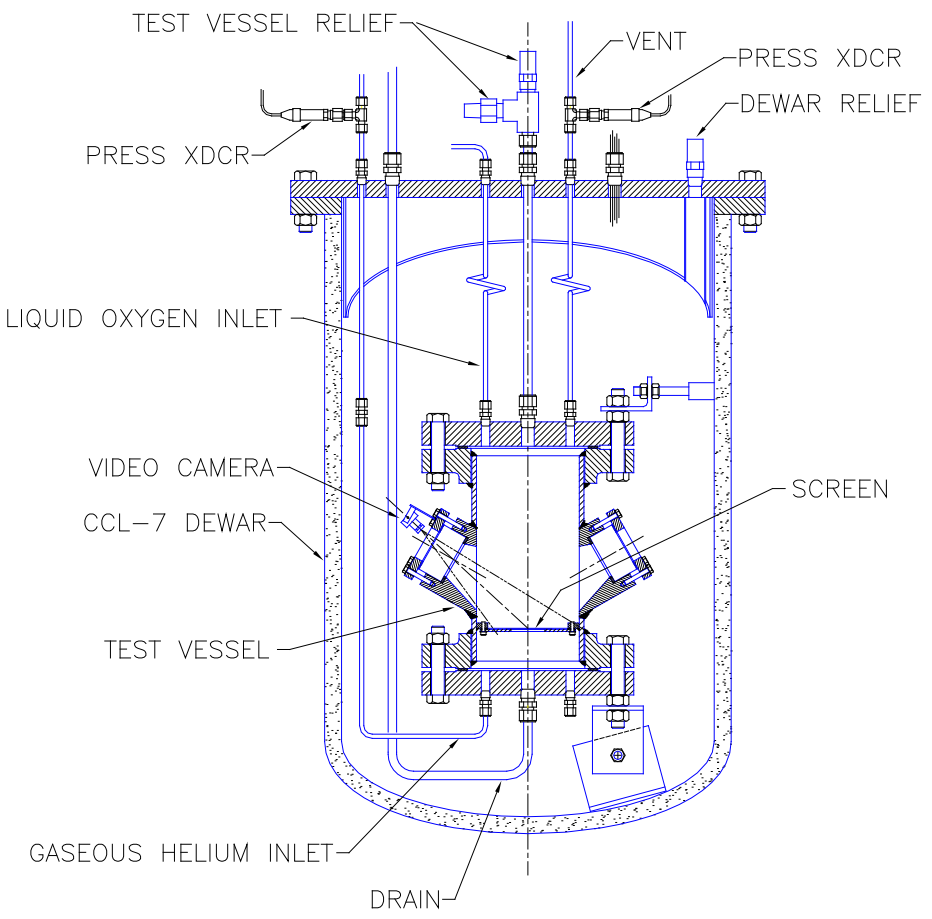

Figure 4: Dewar, CFM Test Tank, and LAD Screen/Cup Assembly

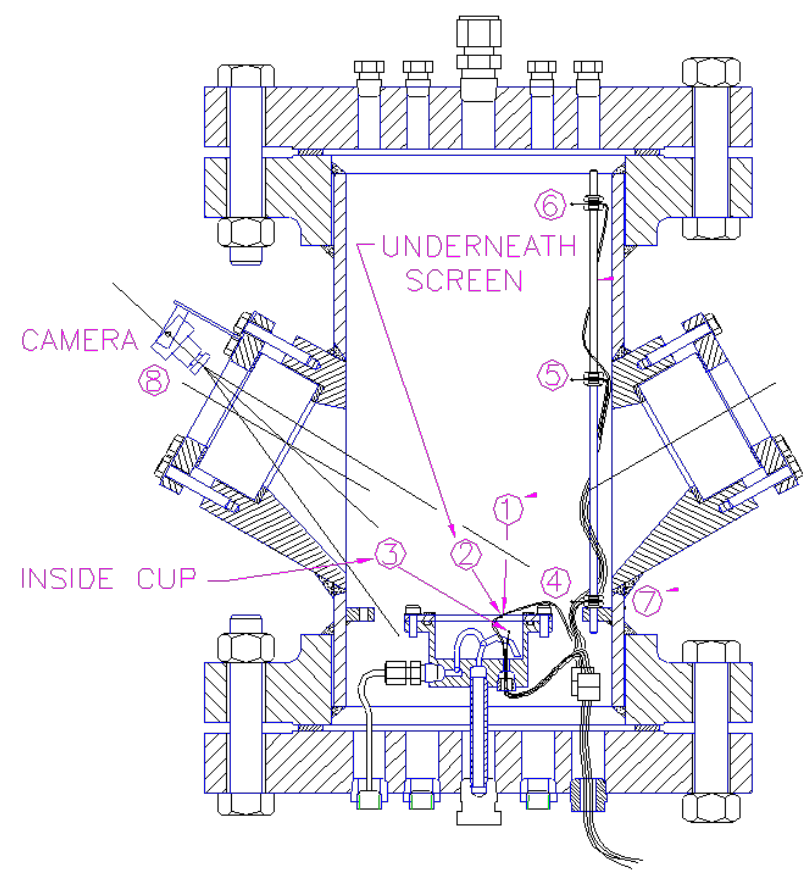

Figure 5: Location of Temperature Measurements Inside CFM Test Tank
CFM test tank was to hold the high pressure liquid cryogen on top of the LAD screen. Since there was no active temperature control of the CFM test tank at cold cryogenic temperatures, the purpose of the dewar was to minimize heat leak into the tank via natural convection between ambient and the CFM test tank. During tests, a full vacuum was pulled on the vacuum jacket of the dewar, while CCL-7 ejectors were used to drop the pressure inside of the receiver dewar to $0.10 \mathrm{kPa}$. While this minimized heat leak into the CFM test tank, there was always a natural tendency for the system to rise in temperature and pressure due to parasitic heat leak during testing. The lid on the dewar was equipped with seven feedthrough ports for vacuum, relief, instrumentation, and electrical wiring.

The CFM test tank was also equipped with several ports on top and below for liquid fill and drain, pressurization, back pressure control, instrumentation, and relief as shown in Figures 4 and 5 . To view the LAD screen, the CFM test tank was equipped with three $7.62 \mathrm{~cm}$ (3.0') OD, $3.81 \mathrm{~cm}$ (1.5') thick quartz windows to

4

American Institute of Aeronautics and Astronautics 
allow optical access to a single charge coupled device (CCD) camera and two light sources. The image of the camera was time stamped, recorded, and sent directly to a monitor inside the cell to provide a real time image of the LAD screen during testing. To prevent condensation of air on the camera at colder receiver dewar temperatures, a heater was mounted to the back of the camera to maintain the camera in its operational temperature band of $4^{\circ} \mathrm{C}-$ $15.5^{\circ} \mathrm{C}\left(40^{\circ} \mathrm{F}-60^{\circ} \mathrm{F}\right)$.

Key temperature instrumentation is outlined in Figure 5 where each number corresponds to the location of a specific silicon diode. Silicon diode-1 (SD1) was mounted directly to the LAD screen on the liquid side of the interface while SD2 was attached to the vapor side located within the cup assembly. Both diodes were directly mounted to the LAD screen using a low thermal conductivity epoxy. SD3 measured the temperature of incoming pressurization gas. SD4 - 6 were located along a vertical rake within the liquid of the test tank and were used to measure both temperature and height of the bulk liquid. The pressure in the ullage space was measured using a 0500 psia pressure transducer while two Setra 205-2 differential pressure transducers (DPT) were used to deduce the liquid head pressure on top of the screen and differential pressure across the LAD screen sample. DPT03 (0 - 30" $\mathrm{H}_{2} 0$ ) measured the pressure at the bottom of the screen/cup assembly with respect to the ullage pressure while DPT01 ( $0-5$ psid) measured the pressure at the bottom of the CFM test tank referencing the ullage pressure. Bubble point was deduced by correcting the DPT03 measurement for head pressure using the DPT01 signal. While the CFM test tank was rated up to $3.45 \mathrm{MPa}$ (500 psia), data collection was limited to $1.725 \mathrm{MPa}$ (250 psia) as limited by the maximum allowable DPT line pressure. All silicon diodes measured temperature to within +/- $0.5 \mathrm{~K}$. The ullage pressure and thus the pressure at the screen was within $+/-0.667$ psia of the measured value. The DPT across the LAD screen sample measured pressure to within $+/-0.042$ in $\mathrm{H}_{2} \mathrm{O}$, which was less than $1 \%$ at the lowest reported bubble point at the highest liquid temperature.

Pressure of the liquid inside the CFM test tank was controlled using a back pressure control valve while temperature of the liquid was primarily controlled by the initial temperature at fill. Colder liquid states were achievable at initial fill while warmer liquid temperatures were achieved by simply allowing the liquid in the LOX supply dewar and/or CFM test tank to warm in time. Gas pressure and flow rate into the LAD screen/cup assembly were controlled manually using a set of low flow control valves to slowly ramp up the pressure. Gaseous helium (GHe) and gaseous oxygen (GOX) were used to pressurize the underside of the LAD screen, and both gases were available via a portable tuber trailer and K-bottles, respectively.

Videos of the LAD screen were recorded to correlate with the time stamped data files to deduce the bubble point. The image was time stamped to a resolution of 0.1 seconds and recorded using a DVD player located inside Cell 7. All data channels were recorded at $2 \mathrm{~Hz}$ using LabVIEW ${ }^{\mathrm{TM}}$ data acquisition system.

\section{Experimental Methodology}

The methodology for conducting a bubble point test is as follows. Before flowing LOX, the LAD screen/cup was pressurized using GHe to prevent flooding of the cup during liquid transfer. Then the hardware was pre-chilled several times using $\mathrm{LN}_{2}$ to condition the system to the desired initial temperature. Liquid oxygen was then transferred into the CFM test tank with the vent valve open from a portable dewar located just outside Cell 7 that was set with its own back pressure control to condition the fluid to the saturation temperature over the pressure range of 0.101 to $1.82 \mathrm{MPa}$.

To conduct a bubble point test, the CFM test tank was isolated from ambient conditions. Measurements would commence if ullage pressure and liquid temperature remained relatively constant. Using a set of metering valves, the pressure underneath the screen was increased relative to the ullage pressure. Eventually the pressure would be sufficient to break through the wetted side of the screen, as indicated in both visual image of a bubble penetrating the screen and a spike in the DPT03 signal. The bubble point was taken as the pressure differential the moment that visible gas or vapor bubbles were observed to penetrate through the screen mesh. The time stamp at breakthrough was noted and compared with the time stamp in the LabVIEW data file to extract the bubble point pressure. Then the pressure underneath the screen was gradually decreased until the screen resealed as indicated in the video file and leveling off in the DPT03 signal. The process was then repeated at different liquid pressures and temperatures. While there may be preferential sites for bubbles breaking through the screen because of small defects in the screen, there was still a fair amount of variability with regards to the location of the bubble penetration point from test to test. Thus bubble points were also repeated at similar conditions to ensure good repeatability and consistency in the data, and to rule out the possibility of these screen defects. Once testing was completed, any residual cryogen was directly vented or drained, and routed to a small flash tank outside of CCL-7. Pressure in the receiver dewar was raised back to ambient. Residual vapor trapped within any of the flow lines was purged using GHe or GN $\mathrm{G}_{2}$. Videos of the LAD screen were then transferred to portable DVDs. 
Prior to this test, bubble points for screen channel LADs were only examined at or slightly below saturated liquid conditions at room pressure. For this test, both subcooled and saturated states at the liquid side screen surface were achievable by pressurizing the ullage space at the top of the CFM test tank or by simply allowing the cryogen to warm in time, respectively. To obtain bubble points in colder liquid temperatures, the system was pre-chilled numerous times before flowing LOX. To obtain bubble points in warmer liquid temperatures, the LOX was preconditioned in its portable dewar and the system was allowed ample time to warm before liquid transfer. To change the pressure of the liquid at a given temperature, the ullage space above the screen sample was pressurized with GHe or GOX. Thus, it was possible to collect bubble points along the saturation curve as well as at various levels of liquid subcooling over the full pressure and temperature range from ambient up to the maximum pressure of 1.79 MPa (250 psia). In this way, both the temperature and pressure dependence of bubble point were independently examined through the use of this flexible system using both pressurization gases.

\section{Results}

Over the course of 8 weeks from February to April 2010, numerous bubble point tests were conducted over the temperature range from 92 to $130 \mathrm{~K}$ and the pressure range from 0.138 to $1.79 \mathrm{MPa}$. Figures $6 \mathrm{a}$ and $6 \mathrm{~b}$ plot the thermodynamic state of the LAD screen during a controlled bubble breakthrough in terms of pressure on top of the LAD screen and the temperature on top of the screen and in the bulk liquid, respectively. As shown, using GHe as a pressurant gas, it was possible to obtain controlled breakthroughs over the full range of conditions. There is little disparity between screen and bulk liquid temperature when reporting the bubble point. However, when using GOX as the pressurant gas, there is considerable difference between reporting bubble breakthrough values using SD1 vs. SD4. The data clustering around the saturation curve indicates that, regardless of the level of subcooling of the liquid in the tank, at breakthrough, the temperature of the liquid/vapor interface within the screen pores tended toward the local saturation temperature based on the pressure at the screen. This implies that the screen was relatively insensitive to changes in the bulk liquid during breakthrough with autogenous (GOX) tank pressurization and relatively sensitive to bulk liquid temperature when using a noncondensible (GHe) pressurization scheme.
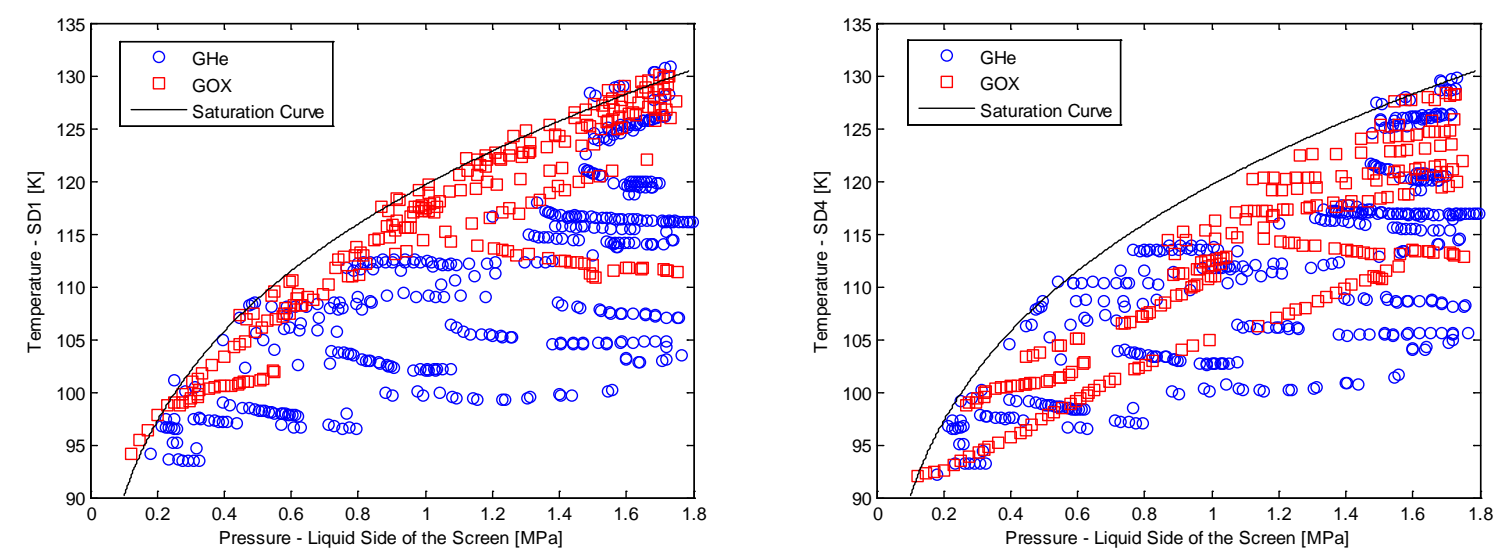

Figure 6: Thermodynamic Conditions at Bubble Breakthrough as a Function of the Pressure on Top of the LAD Screen and a) Temperature of the Liquid Side of the Screen (SD1) and b) Temperature of the Bulk Liquid (SD4)

\section{Discussion of Results}

Figures $7 \mathrm{a}$ and $7 \mathrm{~b}$ plot the experimentally obtained bubble point pressure as a function of the liquid screen side temperature and bulk liquid temperature, respectively, along with the prediction curve based on Equation 1 for zero contact angle. In both cases, the bubble point pressure decreases with increasing liquid temperature, due to decreasing surface tension of the liquid. The model qualitatively tracks this trend but is shown to under predict the data, especially at colder liquid temperatures. At identical liquid temperatures, bubble point pressures using GHe as a pressurant are, on average, higher than those obtained using GOX. The model under predicts the data by as much as $30 \%$ for GHe while better agreement is obtained when using GOX as a pressurant. Since the model generally under predicted GHe data, Equation 1 may be used as a lower bound to predict screen channel LAD performance for a flight system, since the actual breakdown pressure is slightly higher for all temperatures tested here. For a system designer, the data implies that using GHe to pressurize and subcool the LOX during expulsion results in higher 

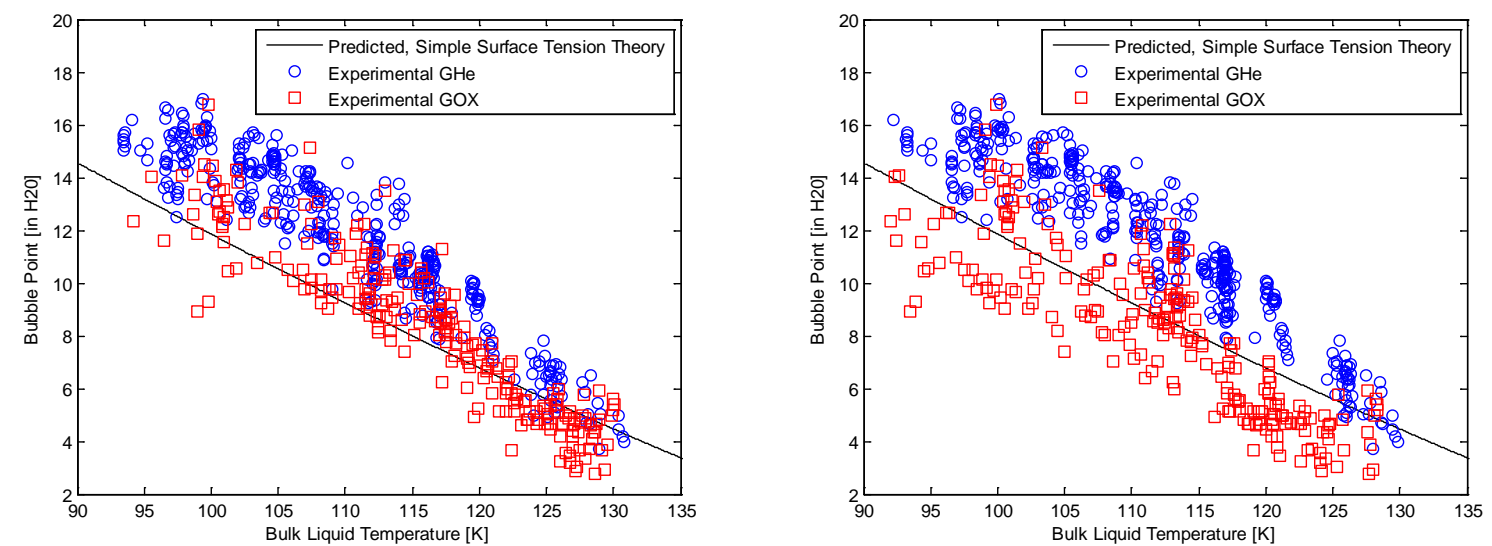

Figure 7: Bubble Point Pressure Reported as a Function of a) Temperature of the Liquid Side of the Screen (SD1) and b) Temperature of the Bulk Liquid (SD4)

margin in the total allowable pressure loss for the LAD system, since the bubble point represents the maximum allowable pressure drop before vapor is ingested into the channel and in the transfer line to the engine.

Comparing Figures $7 \mathrm{a}$ and $7 \mathrm{~b}$, the data and model appear to correlate better when using the liquid screen side temperature over the bulk liquid temperature, especially for GOX pressurization. The scatter in Figure 7b could explain scatter in the data from previous bubble point measurements in subcooled liquid such as Jurns and McQuillen (2008), but temperature sensors at the screen were not available for that measurement. The bulk of the scatter in the data in Figure 7 may be attributed to the fact that bubble points at single temperatures were collected across a range of different pressures. Note that there is more variation in bubble point at colder liquid temperatures, which were achievable under a broad range of pressures, while there is less variation at warmer temperatures, which were only achievable at higher pressures within the facility limits.

This supposed pressure dependence is illustrated in Figure 8. The prediction curve is calculated from surface tension using the saturation temperature based on the pressure at the screen. For all pressures, bubble points using GHe are higher than bubble points using GOX. Elevated

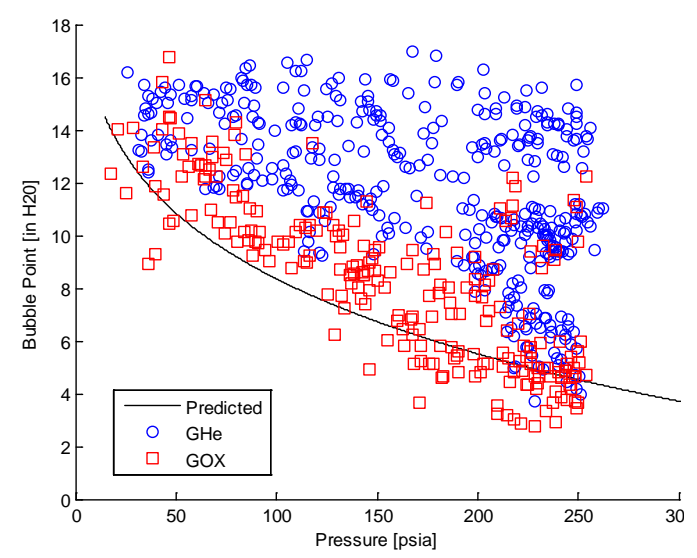

Figure 8: Pressure Dependence of Bubble Point bubble points are achievable using GHe for all pressures (depending on the liquid temperature) while bubble points obtained using GOX fall off with the NBP prediction curve.

Surface tension is generally known to be a function of temperature only, and of the form proposed by Ferguson and Kennedy ${ }^{11}$ :

$$
\sigma=\sigma_{c}\left(1-\frac{T}{T_{C}}\right)^{K}
$$

Incorporating the temperature dependence of Equation 3 into Equation 1 indicates that the bubble point pressure has no pressure dependence. However, during transfer of liquid from the propellant tank through the LAD to the transfer line, the liquid temperature and pressure difference between engine and propellant tank govern the flow rate through the LAD and the amount of vaporization that may occur at the LAD screen. 


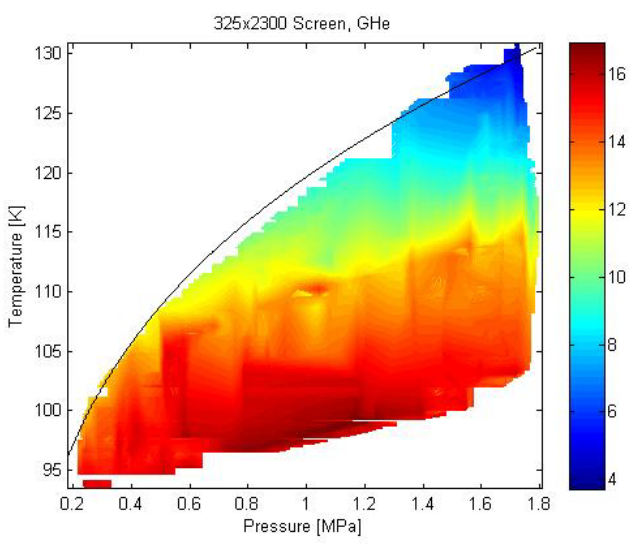

a

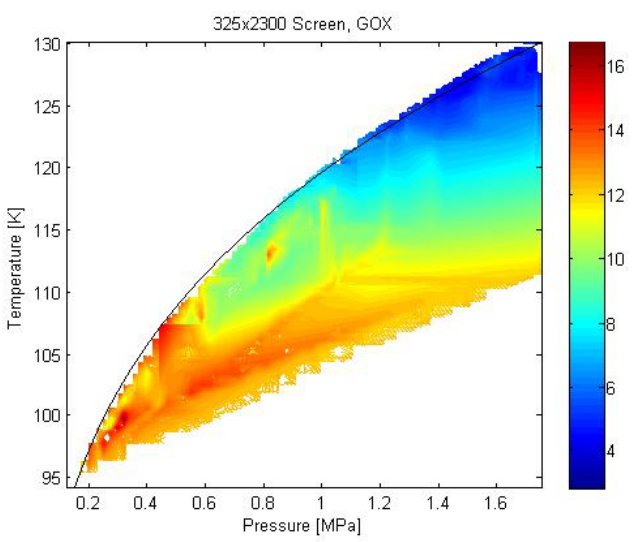

b

Figure 9: Bubble Point for 325 X 2300 screen as a Function of Temperature and Pressure for (a) Gaseous helium as a Pressurant (b) Gaseous Oxygen as a Pressurant. Color lines represent lines of constant bubble point in units of [in $\mathrm{H}_{2} \mathrm{O}$ ].

To investigate this apparent pressure dependence, Figure 9 superimposes the bubble point data as a function of the temperature (SD1) and pressure at the LAD screen on the saturation curve. Thus Figure 9 is a surface plot of Figure 6 where the color scale is used to represent the magnitude of the bubble point. As expected, higher bubble points were obtained at colder liquid temperatures for both pressurization gases. However, there is also a weak secondary effect of pressure on the bubble point, especially as the liquid conditions became more subcooled. The highest bubble points were obtained in the coldest liquid at the highest pressures. Large discrepancies were again observed when using the temperature of the bulk liquid to correlate the data. The implication here is that pressurizing with helium gas buys a system designer margin in temperature or bubble point, while pressurizing with oxygen seems to have a negligible effect.

Closer examination of the temperature measurements at bubble breakthrough revealed some unusual, but not totally unexpected results. Figures $10 \mathrm{a}$ and $10 \mathrm{~b}$ plot the bubble point pressure as a function of the temperature difference across the screen and temperature difference between the liquid screen side temperature and the bulk liquid, respectively. As shown, for both pressurization gases, there is always a positive temperature difference across the screen at breakthrough, as the gas was always slightly warmer than the liquid. Ambient pressurization gas had enough residence time within the dewar walls and piping within the dewar en route to the LAD screen/cup assembly that the gas was nearly at cryogenic temperatures, but the differential at the screen was always positive. Figure 9a shows minimal heat transfers across the screen during breakthrough with GHe, as the temperature difference is no more than $2 \mathrm{~K}$ across the entire range of liquid temperatures.

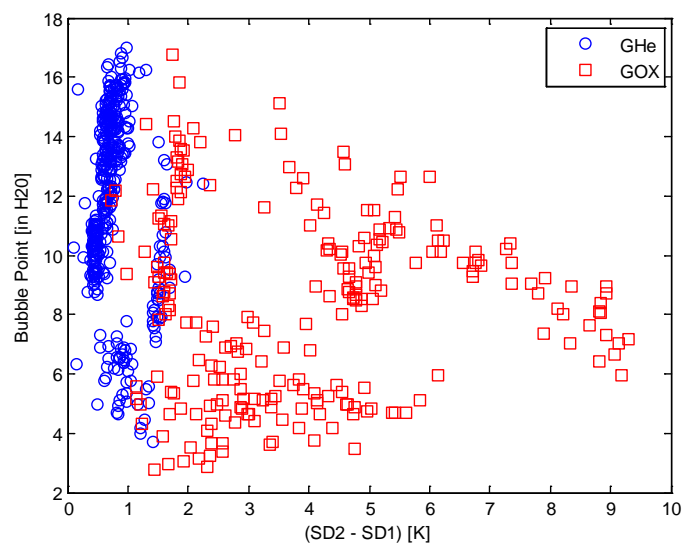

a

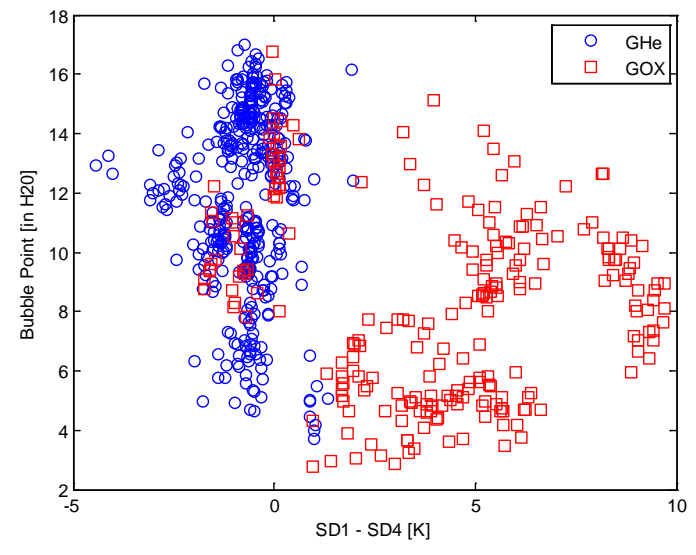

$\mathrm{b}$

Figure 10: Bubble Point as a Function of Temperature Differences (a) Across the Screen, SD2 - SD1, and (b) Within the Liquid Phase from the Screen to the Bulk Liquid Measurement Location, SD1 - SD4 
In contrast, there is a significant amount of heat transfer across the screen when using GOX as the pressurization gas, as temperature differentials are in excess of $9 \mathrm{~K}$. Since higher bubble points were obtained at colder liquid temperatures, it was expected that more heat transfer would occur when the liquid was subcooled but this trend is not apparent in Figure 10a; heat transfer across the screen is relatively constant for GHe and reaches a maximum value in the intermediate bubble point range for GOX. It is evident that pressurization with the vapor causes significantly more heat transfer across the screen than pressurizing with the noncondensible GHe. This is consistent with findings by Sefiane and Ward ${ }^{12}$ who found large temperature differences across a gas-liquid interface where there was mass transfer from one phase to the other.

Examination of Figure 10b indicates that minimal heat transfers between the top of the screen and bulk liquid during breakthrough with GHe. The bulk liquid is shown to be warmer than the top of the screen for the majority of test cases. Meanwhile, there is a considerably large temperature difference (in excess of $9 \mathrm{~K}$ ) between the top of the screen and bulk liquid when using GOX. The bulk liquid is shown to be significantly colder than the top of the screen. This implies that pressurizing with GHe may cause the LOX to evaporate away from the screen, cooling the screen and its immediate vicinity, while pressurizing with GOX may cause rapid condensation of the gas into the liquid, causing the screen and its immediate vicinity to warm. This may partially explain why bubble point pressures in GHe are higher than in GOX. Pressurization with GHe may cause the local temperature at the screen to decrease, increasing the surface tension of the LOX at the screen, thus increasing the bubble point while GOX causes the temperature at the screen to rise, decreasing the surface tension, thus decreasing the bubble point.

To illustrate the 2D trend in Figure 10 on a 3D plot, Figures 11a and 11b plot the bubble point as a function of SD1 as a function of the temperature gradient across the screen at breakthrough for GHe and GOX, respectively. It was initially anticipated that larger temperature differences proportional to the level of subcooling (i.e. the larger the difference between the saturation pressure based on SD1 and the ullage pressure) would exist. Thus it was anticipated that maximum heat transfer across the screen would occur at the coldest liquid temperatures at the highest pressure, and decrease as the liquid temperature approached the local saturation temperature based on the pressure at the LAD screen. However, Figure 11a shows relatively uniform heat transfer across the range of saturated and subcooled liquid states for GHe pressurization, while Figure 11b shows an actual peak in the heat transfer across the screen for GOX pressurization in the range of $110-120 \mathrm{~K}$. Equation 1 predicts very well for bubble points obtained when the liquid temperature is close to the saturation temperature. Therefore it is shown that, for a given screen liquid temperature, that the largest heat transfer across the screen occurred when the temperature at the screen liquid/vapor interface approached the saturation temperature based on the liquid side screen pressure.
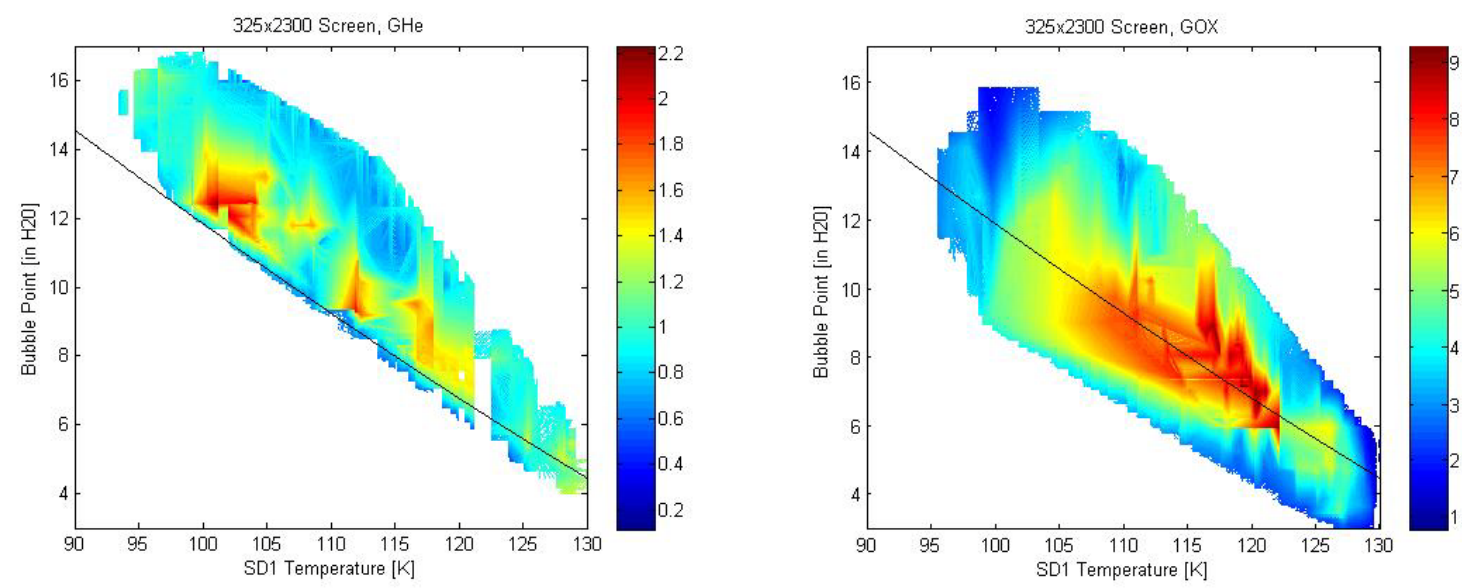

a

b

Figure 11: Temperature Difference across the Screen as a Function of the Liquid Side Screen Temperature and the Bubble Point for (a) Gaseous Helium as a Pressurant, (b) Gaseous Oxygen as a Pressurant. Color lines represent lines of constant temperature gradients across the screen in units of [K].

To further examine this effect, typical temperature and differential pressure across the screen transients are analyzed from initial ramp in pressure until bubble breakthrough, as plotted in Figures 12a and 12b for SD1 - 4 and DPT03 using GHe and GOX, respectively. Snapshot images from the camera are superimposed upon their corresponding time. At bubble breakthrough for the GHe case, the visual imagery is apparent as there is obvious 
mass transfer across the screen that corresponds to the single spike in DPT03 pressure. For this particular run, it is also noted that the temperature difference among all four sensors was less than $2 \mathrm{~K}$.
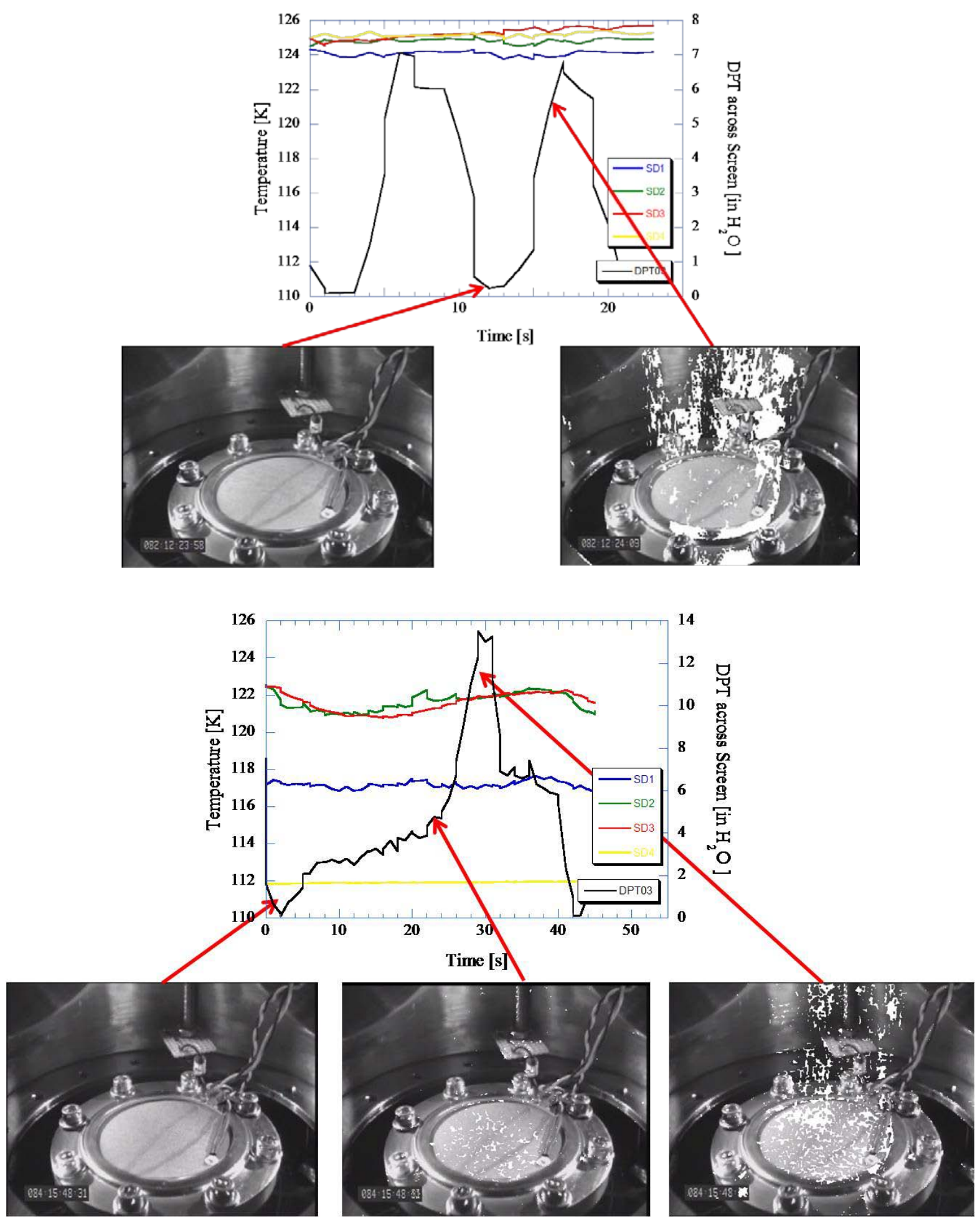

Figure 12: Time Trace of Temperature Sensors and Differential Pressure Transducer with Corresponding Images of Screen for (a) Gaseous Helium as a Pressurant (b) Gaseous Oxygen as a Pressurant 
However, re-evaluating the bubble breakthrough conditions for GOX from the visual imagery was less than certain. Careful study of the GOX video files reveals the possibility of mass transport across the screen before visible bubbles break through the screen. Alternatively, at high heat transfer rates across the screen, due to the rapid condensation of the GOX into the cold LOX bubbles, were not readily detectable after they had risen any appreciable distance from the screen. Image subtraction and enhancement was used to show differences between the screen at initial pressurization and the screen as the test evolved. For Figure 12a, this equates to the visible bubbles. For Figure 12b, this analysis shows the initial thermal distortions at an intermediate time and the bubbles at the end of pressurization. Thus Figure 12b also shows that, during an intermediate time frame, from approximately 5 to 22 seconds, bubbles may have broken through the screen but were immediately condensed into the liquid. While the temperature difference between the gas/vapor phase temperature sensors (SD3 - SD2) was minimal, the temperature difference across the screen was about $4 \mathrm{~K}$. There was an additional 5K.difference between the bulk liquid temperature and the liquid temperature at the screen. Therefore, it is evident that oxygen vapor that initially permeates the screen may initially condense and release latent heat into the surrounding liquid oxygen. Eventually, the local liquid temperature at the screen warms, and bubbles break through. In this way, there is mass transport and minimal heat transport across the screen at bubble breakthrough using GHe and a considerable amount of mass and heat transport across the screen when using GOX.

\section{Plans}

Future plans include the analysis of bubble point data for the 200 x1400 screen in LOX as well analysis of bubble point data for the 325x2300 screen in LN2. Since LN2 has different thermophysical properties than LOX, it will be interesting to see if the trends outlined in this work match in a different cryogenic fluid. Testing is currently ongoing using $\mathrm{LCH}_{4}$ at the same range of elevated temperatures and pressures. This data will also be analyzed and compared to trends outlined in this work.

\section{Conclusion}

As a result of this study, it is shown that better agreement between data and theory is obtained when using the liquid screen side interfacial temperature to report the bubble point for a screen channel LAD. This negates the previous assumption that the bubble point scales with the bulk liquid temperature. Bubble point predictions based on simple surface tension theory hold for saturated liquid states but fail to predict for subcooled liquid states. Across the full temperature range, bubble point is shown to be a strong function of liquid temperature that qualitatively scales with the surface tension of the liquid.

For all pressures, the bubble point is higher than the predicted value when using GHe to pressurize the system. Minimal heat transfer across the screen occurs as warmer GHe may evaporate a fraction of the liquid at the screen, cooling the screen, and increasing the local surface tension and bubble point. This has implications in the design of LADs for in-space cryogenic propellant systems since pressurization with GHe yields a bigger margin in the total allowable pressure drop in a flight screen channel LAD system during fluid transfer from the propellant tank to the engine.

Meanwhile, good agreement exists between theoretical predictions and experimentally obtained bubble points using GOX. Considerable heat transfer across the screen occurs prior to and during breakthrough, which may cause warm GOX bubbles to condense into the liquid, warming the screen, and decreasing the local surface tension and bubble point pressure. As is evident from image subtraction techniques, initial heat transfer across the screen associated with condensation of vapor during the initial pressure ramp is followed by the eventual breakthrough of a visible bubble. Therefore the supposed pressure dependence simply modifies the temperature at the screen through added heating or cooling of the local liquid oxygen and screen through variable condensation and evaporation at breakthrough.

\section{Acknowledgments}

This work was funded by the Cryogenic Fluid Management Project at NASA Glenn Research Center under the auspices of the Exploration Systems Mission Directorate at NASA Headquarters. The authors would like to thank the operations team and research support staff at CCL-7 for their assistance during planning and testing phases. 


\section{References}

1 Paynter, H. L., “Acquisition/Expulsion System for Earth Orbital Propulsion System, Vol. III, Cryogenic Test,” Martin Marietta Corp., Denver, Colo., MCR-73-97, 1973.

2 Cady, E.C. "Study of Thermodynamic Vent and Screen Baffle Integration for Orbital Storage and Transfer of Liquid Hydrogen - Final Report” NASA-CR-134482, 1973.

3 Cady, E.C., "Design and Evaluation of Thermodynamic Vent/Screen Baffle Cryogenic Storage System,” NASA-CR134810, 1975.

4 Cady, E.C., "Effect of Transient Liquid Flow on Retention Characteristics of Screen Acquisition Systems,” NASA-CR135218, 1977.

5 Chato, D.J. and Kudlac, M.T., "Screen Channel Liquid Acquisition Devices for Cryogenic Propellants,” AIAA 75-1196, 38th Joint Propulsion Conference and Exhibit Indianapolis, IN, July 7-10, 2002.

6 Kudlac, M.T. and Jurns, J.M, “Screen Channel Liquid Acquisition Devices for Liquid Oxygen,” AIAA-2006-5054, 42nd Joint Propulsion Conference, Sacramento, CA, July 9 - 12, 2005.

7 Jurns, J.M., McQuillen, J.B., Gaby, J.D., and Sinacore S.A., "Bubble Point Measurements with Liquid Methane of a Screen Channel Capillary Liquid Acquisition Device” 54th JANNAF Conference, Denver, CO, May 14 - 17, 2007.

8 Bretherton, F.P. “The Motion of Long Bubbles in Tubes” Journal of Fluid Mechanics 10, 166 - 188, 1961.

9 Jurns, J.M. and McQuillen, J.B., “Liquid Acquisition Device Testing with Sub-cooled Liquid Oxygen” AIAA-20084943 44th Joint Propulsion Conference and Exhibit, Hartford, CT, July 21 - 23, 2008.

10 Roder, H. M. and Weber, L. A. “ASRDI Oxygen Technology Survey. Volume 1: Thermophysical Properties,” NASA SP 3071, 1972.

11 Ferguson, A. and Kennedy, S. J., Trans. Faraday Soc., 32, 1474, 1936.

12 Sefiane, K. and Ward, C. A., "Recent advances on thermocapillary flows and interfacial conditions during the evaporation of liquids,” Advances in Colloid and Interface Science 134-135, 201-223, 2007.

13 Van Dyke, M. "Identification of Influential Factors for Liquid Acquisition Device Designs" AIAA-98-3198, 1998. 